\title{
An Applications of Information Systems on Macro-Economic Climate Index of China ${ }^{\#}$
}

\author{
Mei He ${ }^{1}$, Xun Ge 2* $^{*}$ \\ ${ }^{1}$ School Mathematical Science, Huaiyin Normal University, Huaian, China \\ ${ }^{2}$ College of Zhangjiagang, Jiangsu University of Science and Technology, Zhangjiagang, China \\ E-mail: hemei94@126.com, ${ }^{*}$ zhugexun@163.com \\ Received January 11, 2011; revised March 2, 2011; accepted March 21, 2011
}

\begin{abstract}
Recently, National Bureau of Statistics of China has released macro-economic climate index of China from 2009-02 to 2010-05.Based on these indices, we establish an information system. In this information system, monitoring signal is taken as a decision attribute and coincident index, leading index, lagging index are taken as condition attributes. We use rough-set theory to investigate the importance of each condition attribute with respective to decision attribute and the strength of each condition attribute supporting decision attribute. Results of this investigation will be helpful for Chinese government to make active macro-economic policy and to maintain the steady and relatively fast development of Chinese economy.
\end{abstract}

Keywords: Information System, Condition Attribute, Decision Attribute, Macro-Economic Climate Index, Monitoring Signal, Coincident Index, Leading Index, Lagging Index

\section{Introduction}

The global financial crisis that started two years ago has taken its toll on the world economy on an unprecedented scale. Ow to make world economy recovery as soon as possible? Art of the recent optimism in world markets rests on the belief that China's iscal-stimulus package is boosting its economy and that GDP growth ould come close to the government's target of $8 \%$ this year. China has not only accomplished considerable fiscal and monetary easing, but also Beijing is passing on some of that boost to the rest of the world. Recently, National Bureau of Statistics of China has released the following table of macro-economic climate index of China from 2009-02 to 2010-05 (Table 1) [1]. For this table, the compilation methods and summary analysis can be found at www. cemac.org, and following remark is given at first.

Remark 1. 1) The monitoring signal shows state of the economic operation.

2) The coincident index is the index reflecting the current basic trend of the economy, and it is calculated with the following data:

(a) industrial production;

"Project supported by the National Nature Science Foundation of China (No. 10971185 and 11061004). And Natural Science Research Project of Ordinary Universities in Jiangsu (08KJB110002). *Corresponding author
Table 1. Macro-economic climate index of China from 2009-02 to 2010-05, (year $1996=100)$.

\begin{tabular}{ccccc}
\hline Date & $\begin{array}{c}\text { Monitoring } \\
\text { Signal }\end{array}$ & $\begin{array}{c}\text { Coincident } \\
\text { Index }\end{array}$ & $\begin{array}{c}\text { Leading } \\
\text { Index }\end{array}$ & $\begin{array}{c}\text { Lagging } \\
\text { Index }\end{array}$ \\
\hline 2009.02 & 76.0 & 94.0 & 99.0 & 92.7 \\
2009.03 & 82.0 & 94.5 & 100.0 & 91.2 \\
2009.04 & 78.0 & 94.9 & 101.0 & 90.7 \\
2009.05 & 84.0 & 95.4 & 101.9 & 90.0 \\
2009.06 & 86.7 & 96.0 & 102.6 & 89.5 \\
2009.07 & 94.0 & 96.6 & 103.4 & 89.6 \\
2009.08 & 96.7 & 97.3 & 104.1 & 90.0 \\
2009.09 & 103.3 & 98.2 & 105.0 & 90.8 \\
2009.10 & 110.7 & 99.5 & 105.7 & 92.0 \\
2009.11 & 117.3 & 100.4 & 105.4 & 93.2 \\
2009.12 & 116.7 & 102.1 & 105.4 & 93.8 \\
2010.01 & 114.0 & 103.1 & 104.7 & 95.0 \\
2010.02 & 104.7 & 103.9 & 104.7 & 95.8 \\
2010.03 & 106.0 & 104.1 & 105.0 & 96.3 \\
2010.04 & 107.3 & 104.0 & 104.4 & 96.3 \\
2010.05 & 114.0 & 103.7 & 103.4 & 97.3 \\
\hline
\end{tabular}


b) employment;

c) social demands (including investment, consumption and foreign trade);

d) social incomes (including the government taxes, profits of enterprises and income of residents).

3) The leading index is calculated with a group of leading indicators, which take a lead before the coincident index, and is used for forecasting the future economic trend.

4) The lagging index is calculated with the lagging indicators, which lag behind the coincident index, and is mainly used for confirming the peak and valley of the economic cycle.

Naturally, we are interested in some relations between monitoring signal and its impacting factors (coincident index, leading index, lagging index) in the above table. In macro-economic climate index of China, monitoring signal of each month, as well as the associated its impacting factors are uncertain decisions, so it is unlikely to be appropriate to use traditional analytic methods (e.g. synthesis, appraisal, stratification and estimate of probability). However rough-set theory, which is a logicmathematical method proposed by Z. Pawlak, has shown to be an effective tool in analyzing this type of issues [2-5]. In recent years, this theory has been widely implemented in the many fields of natural science and societal science [6-9,10,11-13]. In this paper, we establish an information system based on the above table. In this information system, monitoring signal is taken as a decision attribute and coincident index, leading index, lagging index are taken as condition attributes. We use rough-set theory to investigate the importance of each condition attribute with respective to decision attribute and the strength of each condition attribute supporting decision attribute. Results of this investigation will be helpful for Chinese government to make active macro-economic policy and to maintain the steady and relatively fast development of Chinese economy.

\section{Propaedeutics}

Propaedeutics in this section belongs to Z. Pawlak [2-5, 13]. $B$.

Remark 2. 1) For a set $B,|B|$ denotes the cardinal of

2) For a family of sets

$$
\begin{aligned}
& F_{1}, F_{2}, \cdots, F_{k}, \wedge\left\{F_{i}: i=1,2, \cdots, k\right\} \\
& =\left\{\cap\left\{\mathrm{F}_{i}: i=1,2, \cdots, k\right\}: \mathrm{F}_{i} \in F_{i}, i=1,2, \cdots, k\right\}
\end{aligned} .
$$

3) Let $R$ be an equivalence relation on a set $U$. $U / R$ denotes the family consisting of all equivalence classes with respect to $R$ and $[u]$ denotes the equivalence class with respect to $R$ containing $u \in U$.
4) Let $R$ be a family of equivalence relations on $U$. Then $\wedge\{U / \mathrm{R}: \mathrm{R} \in R\}$ is a partition of $U$ and is denoted by $U / \mathrm{R}$. The equivalence relation induced by $U / \mathrm{R}$ is also denoted by $R$.

Definition 1. $S=(U, A, V, f)$ is called an information system.

1) $U$, a nonempty finite set, is called the universe of discourse.

2) $A=C \cup D$ is a finite set of attributes, where $C$ and $D$ are disjoint nonempty sets of condition attributes and decision attributes respectively.

3) $f: U \times A \rightarrow V$ is an information function.

4) $V=\bigcup\left\{V_{\alpha}: \alpha \in A\right\}$, where $V_{\alpha}=\{f(u, \alpha): u \in U\}$.

Remark 3. An information system $S=(U, A, V, f)$ can be expressed a date table, which is called decision table, whose columns are labeled by elements of $A$, rows are labeled by elements of $U$, and $f(u, a)$ lies in the cross of the row labeled by $u$ and the column labeled by $a$.

Definition 2. Let $S=(U, C \cup D, V, f)$ be an information system.

1) For $a \in C \cup D$, we define an equivalence relation " " on $U$ as follows:

$$
u_{i} \sim u_{j} \Leftrightarrow f\left(u_{i}, a\right)=f\left(u_{j}, a\right) .
$$

U/a denotes the family consisting of all equivalence classes with respect to " $\sim$ ".

2) For $B \subset C \bigcup D, \wedge\{U / b: b \in B\}$ is a partition of $U$, which is denoted $U / B$. The equivalence relation induced by $U / B$ is also denoted by $B$.

Definition 3 Let $R$ be an equivalence relation on an universe $U$ of discourse, and $X \subset U$. Put $\underline{R}(X)=$ $\cup\{[u] \mid[u] \in U / R,[u] \subset X\} \cdot \underline{R}(X)$ is called lower approximation of $X$.

\section{Decision Table}

In this section, we establish an information system $S=(U, C \cup D, V, f)$ for our investigation, which is obtained by transforming the table of macro-economic climate index of China from 2009-02 to 2010-05 and includes all information we need in this investigation.

Remark 4. Put

$$
\begin{aligned}
U= & \left\{u_{1}, u_{2}, u_{3}, u_{4}, u_{5}, u_{6}, u_{7}, u_{8}, u_{9},\right. \\
& \left.u_{10}, u_{11}, u_{12}, u_{13}, u_{14}, u_{15}, u_{16}\right\}
\end{aligned}
$$

where

$$
u_{1}, u_{2}, u_{3}, u_{4}, u_{5}, u_{6}, u_{7}, u_{8}, u_{9}, u_{10}, u_{11}, u_{12}, u_{13}, u_{14}, u_{15}, u_{16}
$$

denote sixteen months from 2009-02 to 2010-05 in turn. $U$ is the universe of discourse in $S=(U, C \cup D, V, f)$.

Remark 5. Put $D=\{d\}$, where $d$ denotes monitoring signal. $D$ is the set of a decision attribute in 
$S=(U, C \cup D, V, f)$.

Remark 6. Put $C=\left\{c_{1}, c_{2}, c_{3}\right\}$, where $c_{1}, c_{2}, c_{3} d e-$ note coincident index, leading index, lagging index respectively. $C$ is the set of three condition attributes in $S=(U, C \cup D, V, f)$.

Remark 7. $f$ and $V$ in $S=(U, C \cup D, V, f)$ are given as Definition 1 and Remark 3.

This information system $S=(U, C \cup D, V, f)$ is expressed by the following decision table. (Table 2)

Remark 8. Monitoring signals are divided three levels: cooling, stable and heating.

1) $d_{1}$ indicates monitoring signal less than 95.0.

2) $d_{2}$ indicates monitoring signal between 95.0 and 110.0.

3) $d_{3}$ indicates monitoring signal more than 110.0 .

Remark 9. Coincident Indices are divided three levels: cooling, stable and heating.

1) $c_{11}$ indicates coincident index less than 97.0.

2) $c_{21}$ indicates coincident index between 97.0 and 101.0.

3) $c_{31}$ indicates coincident index more than 101.0.

Remark 10. Leading Indices are divided three levels: cooling, stable and heating.

1) $c_{12}$ indicates leading index less than 103.0.

2) $c_{22}$ indicates leading index between 103.0 and 104.9.

3) $c_{32}$ indicates leading index more than 104.9.

Table 2. Decision table.

\begin{tabular}{lllll}
\hline$U$ & $d$ & $c_{1}$ & $c_{2}$ & \\
\hline$u_{1}$ & $d_{1}$ & $c_{11}$ & $c_{12}$ & $c_{23}$ \\
$u_{2}$ & $d_{1}$ & $c_{11}$ & $c_{12}$ & $c_{23}$ \\
$u_{3}$ & $d_{1}$ & $c_{11}$ & $c_{12}$ & $c_{13}$ \\
$u_{4}$ & $d_{1}$ & $c_{11}$ & $c_{12}$ & $c_{13}$ \\
$u_{5}$ & $d_{1}$ & $c_{11}$ & $c_{12}$ & $c_{13}$ \\
$u_{6}$ & $d_{1}$ & $c_{11}$ & $c_{22}$ & $c_{13}$ \\
$u_{7}$ & $d_{2}$ & $c_{21}$ & $c_{22}$ & $c_{13}$ \\
$u_{8}$ & $d_{2}$ & $c_{21}$ & $c_{32}$ & $c_{13}$ \\
$u_{9}$ & $d_{3}$ & $c_{21}$ & $c_{32}$ & $c_{23}$ \\
$u_{10}$ & $d_{3}$ & $c_{21}$ & $c_{32}$ & $c_{23}$ \\
$u_{11}$ & $d_{3}$ & $c_{31}$ & $c_{32}$ & $c_{23}$ \\
$u_{12}$ & $d_{3}$ & $c_{31}$ & $c_{22}$ & $c_{33}$ \\
$u_{13}$ & $d_{2}$ & $c_{31}$ & $c_{22}$ & $c_{33}$ \\
$u_{14}$ & $d_{2}$ & $c_{31}$ & $c_{32}$ & $c_{33}$ \\
$u_{15}$ & $d_{2}$ & $c_{31}$ & $c_{22}$ & $c_{33}$ \\
$u_{16}$ & $d_{3}$ & $c_{31}$ & $c_{22}$ & $c_{33}$ \\
\hline
\end{tabular}

Remark 11. Lagging Indices are divided three levels: cooling, stable and heating.

1) $c_{13}$ indicates lagging index less than 91.0.

2) $c_{23}$ indicates lagging index between 91.0 and 94.0.

3) $c_{33}$ indicates lagging index more than 94.0.

By some simple operations, we obtain the following related partitions of $U$.

Proposition 1. The following are some related partitions of $U$.

1) $U / d=\left\{\left\{u_{1}, u_{2}, u_{3}, u_{4}, u_{5}, u_{6}\right\},\left\{u_{7}, u_{8}, u_{13}, u_{14}, u_{15}\right\}\right.$,

$$
\left\{u_{9}, u_{10}, u_{11}, u_{12}, u_{16}\right\} \text {. }
$$

2) $U / c_{1}=\left\{\left\{u_{1}, u_{2}, u_{3}, u_{4}, u_{5}, u_{6}\right\},\left\{u_{7}, u_{8}, u_{9}, u_{10}\right\}\right.$,

$$
\left.\left\{u_{11}, u_{12}, u_{13}, u_{14}, u_{15}, u_{16}\right\}\right\} \text {. }
$$

3) $U / c_{2}=\left\{\left\{u_{1}, u_{2}, u_{3}, u_{4}, u_{5}\right\},\left\{u_{6}, u_{7}, u_{12}, u_{13}, u_{15}, u_{16}\right\}\right.$,

$$
\left.\left\{u_{8}, u_{9}, u_{10}, u_{11}, u_{14}\right\}\right\} \text {. }
$$

4) $U / c_{3}=\left\{\left\{u_{3}, u_{4}, u_{5}, u_{6}, u_{7}, u_{8}\right\},\left\{u_{1}, u_{2}, u_{9}, u_{10}, u_{11}\right\}\right.$,

$$
\left.\left\{u_{12}, u_{13}, u_{14}, u_{15}, u_{16}\right\}\right\} \text {. }
$$

5) $U / C=\left\{\left\{u_{1}, u_{2}\right\},\left\{u_{3}, u_{4}, u_{5}\right\},\left\{u_{6}\right\},\left\{u_{7}\right\},\left\{u_{8}\right\}\right.$,

$$
\left\{u_{9}, u_{10}\right\},\left\{u_{11}\right\},\left\{u_{12}, u_{13}, u_{15}, u_{16}\right\},\left\{u_{14}\right\} .
$$

6) $U /\left\{c_{2}, c_{3}\right\}=\left\{\left\{u_{1}, u_{2}\right\},\left\{u_{3}, u_{4}, u_{5}\right\},\left\{u_{6}, u_{7}\right\},\left\{u_{8}\right\}\right.$,

$$
\left.\left\{u_{9}, u_{10}, u_{11}\right\},\left\{u_{12}, u_{13}, u_{15}, u_{16}\right\},\left\{u_{14}\right\}\right\} .
$$

7) $U /\left\{c_{1}, c_{3}\right\}=\left\{\left\{u_{1}, u_{2}\right\},\left\{u_{3}, u_{4}, u_{5}, u_{6}\right\},\left\{u_{7}, u_{8}\right\}\right.$,

$$
\left\{u_{9}, u_{10}\right\}\left\{u_{11}\right\},\left\{u_{12}, u_{13}, u_{14}, u_{15}, u_{16}\right\} .
$$

8) $U /\left\{c_{1}, c_{2}\right\}=\left\{\left\{u_{1}, u_{2}, u_{3}, u_{4}, u_{5}\right\},\left\{u_{6}\right\},\left\{u_{7}\right\}\right.$,

$$
\left.\left\{u_{8}, u_{9}, u_{10}\right\},\left\{u_{11}, u_{14}\right\},\left\{u_{12}, u_{13}, u_{15}, u_{16}\right\}\right\} .
$$

\section{Importance}

Definition 4. Let $R$ be a family of equivalence relations on $U$ and $R^{\prime} \subset R$. Let $Q$ be an equivalence relation on $U$.

1) Put $\operatorname{pos}_{R}(Q)=\bigcup\{\underline{R}(X): X \in U / Q\}$.

$\operatorname{pos}_{R}(Q)$ is called positive region of $Q$ with respect to $R$.

2) Put $\gamma_{R}(Q)=\frac{\left|\operatorname{pos}_{R}(Q)\right|}{|U|}$.

$\gamma_{R}(Q)$ is called dependable degree of $Q$ with respect to $R$.

3) Put $\sigma_{R Q}\left(R^{\prime}\right)=\gamma_{R}(Q)-\gamma_{R-R^{\prime}}(Q)$. $Q$.

$\sigma_{R Q}\left(R^{\prime}\right)$ is called importance of $R^{\prime}$ with respect to

Remark 12. For information system $S=(U, C \cup D, V, f)$, let $c \in C . P u t$ 


$$
\sigma_{C D}(\{c\})=\gamma_{C}(D)-\gamma_{C-\{c\}}(D) .
$$

According to Z. Pawlak rough-set theory, $\sigma_{C D}(\{c\})$ is the importance of condition attribute $c$ with respect to decision attribute $d[13]$.

By Proposition 1 and Definition 4(1), we have the following proposition by some simple operations.

Lemma 1. The following hold.

1) $\operatorname{pos}_{C}(D)$

$$
=\left\{u_{1}, u_{2}, u_{3}, u_{4}, u_{5}, u_{6}, u_{7}, u_{8}, u_{9}, u_{10}, u_{11}, u_{14}\right\} .
$$

2) $\operatorname{pos}_{\left\{c_{2}, c_{3}\right\}}(D)=\left\{u_{1}, u_{2}, u_{3}, u_{4}, u_{5}, u_{8}, u_{9}, u_{10}, u_{11}, u_{14}\right\}$.

3) $\operatorname{pos}_{\left\{c_{1}, c_{3}\right\}}(D)$

$$
=\left\{u_{1}, u_{2}, u_{3}, u_{4}, u_{5}, u_{6}, u_{7}, u_{8}, u_{9}, u_{10}, u_{11}\right\} .
$$

4) $\operatorname{pos}_{\left\{c_{1}, c_{2}\right\}}(D)=\left\{u_{1}, u_{2}, u_{3}, u_{4}, u_{5}, u_{6}, u_{7}\right\}$.

By Lemma 1 and Definition 4(2), we have the following proposition by some simple operations.

Lemma 2. The following hold.

1) $\gamma_{C}(D)=\frac{\left|\operatorname{pos}_{C}(D)\right|}{|U|}=\frac{12}{16}=0.750$.

2) $\gamma_{\left\{c_{2}, c_{3}\right\}}(D)=\frac{\left|\operatorname{pos}_{\left\{c_{2}, c_{3}\right\}}(D)\right|}{|U|}=\frac{10}{16}=0.625$.

3) $\gamma_{\left\{c_{1}, c_{3}\right\}}(D)=\frac{\left|\operatorname{pos}_{\left\{c_{1}, c_{3}\right\}}(D)\right|}{|U|}=\frac{11}{16}=0.688$.

4) $\gamma_{\left\{c_{1}, c_{2}\right\}}(D)=\frac{\left|\operatorname{pos}_{\left\{c_{1}, c_{2}\right\}}(D)\right|}{|U|}=\frac{7}{16}=0.438$.

By Lemma 2 and Remark 12, we have the following proposition by some simple operations, which gives the importance of condition attribute $c \in C$ with respect to decision attribute $d$.

Proposition 2. The following hold.

$$
\begin{aligned}
& \text { 1) } \sigma_{C D}\left(\left\{c_{1}\right\}\right)=\gamma_{C}(D)-\gamma_{\left\{c_{2}, c_{3}\right\}}(D) \\
& =0.750-0.625=0.125 \text {. } \\
& \text { 2) } \sigma_{C D}\left(\left\{c_{2}\right\}\right)=\gamma_{C}(D)-\gamma_{\left\{c_{1}, c_{3}\right\}}(D) \\
& =0.750-0.688=0.062 \text {. } \\
& \text { 3) } \sigma_{C D}\left(\left\{c_{3}\right\}\right)=\gamma_{C}(D)-\gamma_{\left\{c_{1}, c_{2}\right\}}(D) \\
& =0.750-0.438=0.312 \text {. }
\end{aligned}
$$

Remark 13. By Remark 12 and Proposition 2, we have the following conclusions.

1) Coincident index (with respect to monitoring signal) is the less important than lagging index and more important than leading index (the importance is 0.125 ).

2) Leading index (with respect to monitoring signal) is the least important in all condition attributes (the importance is 0.062 ).
3) Lagging index (with respect to monitoring signal) is the most important in all condition attributes (the importance is 0.312 ).

\section{Support}

Definition 5. For information system $S=(U, C \cup D, V, f)$, let $W \subset U$ and $c \in C$.

1) Put $S_{C}(W)=\bigcup\{[u]:[u] \in U / c$ and $[u] \subset W\}$.

$S_{C}(W)$ is called a support subset of $W$ with respect to condition attribute $c$.

2) Put $\operatorname{spt}_{c}(W)=\frac{\left|S_{c}(W)\right|}{|U|}$.

$\operatorname{spt}_{c}(W)$ is called the support degree of $W$ with respect to condition attribute $c$.

3) Put $S_{c}(d)=\bigcup\left\{S_{c}(W): W \in U / d\right\}$.

$S_{c}(d)$ is called a support subset of decision attribute $d$ with respect to condition attribute $c$.

Remark 14. For information system

$$
S=(U, C \cup D, V, f) \text {, let } c \in C . P u t . \quad \operatorname{spt}_{c}(d)=\frac{\left|S_{c}(d)\right|}{|U|}
$$

According to Z. Pawlak rough-set theory, $\operatorname{spt}_{c}(d)$ reflects the strength of condition attribute $c$ supporting decision attribute $d$ [13].

Remark 15. Put $W_{1}=\left\{u_{1}, u_{2}, u_{3}, u_{4}, u_{5}, u_{6}\right\}$, $W_{2}=\left\{u_{7}, u_{8}, u_{13}, u_{14}, u_{15}\right\}, W_{3}=\left\{u_{9}, u_{10}, u_{11}, u_{12}, u_{16}\right\}$.Then $U / d=\left\{W_{1}, W_{2}, W_{3}\right\}$.

The following lemma is known [9].

Lemma 3. For information system $S=(U, C \cup D, V, f)$, let $c \in C$. Then the following hold.

1) $S_{c}(d)=S_{c}\left(W_{1}\right) \cup S_{c}\left(W_{2}\right) \cup S_{c}\left(W_{3}\right)$.

2) $S_{c}\left(W_{i}\right) \cap S_{c}\left(W_{j}\right)=\varnothing$ for all $i, j=1,2,3$ and $i \neq j$.

3) $s p t_{c}(d)=s p t_{c}\left(W_{1}\right)+s p t_{c}\left(W_{2}\right)+s p t_{c}\left(W_{3}\right)$.

By Proposition 1, Definition 5(1) and Remark 15, we have the following lemma by some simple operations.

Lemma 4. The following hold.

1) $S_{c_{1}}\left(W_{1}\right)=\left\{u_{1}, u_{2}, u_{3}, u_{4}, u_{5}, u_{6}\right\}, S_{c_{1}}\left(W_{2}\right)$

$$
=S_{c_{1}}\left(W_{3}\right)=\varnothing \text {. }
$$

2) $S_{c_{2}}\left(W_{1}\right)=\left\{u_{1}, u_{2}, u_{3}, u_{4}, u_{5}\right\}, S_{c_{2}}\left(W_{2}\right)$

$$
=S_{c_{2}}\left(W_{3}\right)=\varnothing \text {. }
$$

3) $S_{c_{3}}\left(W_{1}\right)=S_{c_{3}}\left(W_{2}\right)=S_{c_{3}}\left(W_{3}\right)=\varnothing$.

By Definition 5(2) and Lemma 4, we have the following lemma. 
Lemma 5. The following hold.

1) $s p t_{c_{1}}\left(W_{1}\right)=\frac{\left|S_{c_{1}}\left(W_{1}\right)\right|}{|U|}=\frac{6}{16} ; \quad s p t_{c_{1}}\left(W_{i}\right)=\frac{\left|S_{c_{1}}\left(W_{i}\right)\right|}{|U|}$ $=\frac{0}{16}=0 ;$ for $i=2,3$.

2) $s p t_{c_{2}}\left(W_{1}\right)=\frac{\left|S_{c 2}\left(W_{1}\right)\right|}{|U|}=\frac{5}{16} ; s p t_{c_{2}}\left(W_{i}\right)=\frac{\left|S_{c_{2}}(W)_{i}\right|}{|U|}$ $=\frac{0}{16}=0 ;$ for $i=2,3$.

3) $\operatorname{spt}_{c_{3}}\left(W_{i}\right)=\frac{\left|S_{c_{3}}\left(W_{i}\right)\right|}{|U|}=\frac{0}{16}=0$ for $i=1,2,3$.

By Lemma 3(3), Lemma 5 and Remark 14, we have the following proposition, which gives the strength of condition attribute $c \in C$ supporting decision attribute $d$.

Proposition 3. The following hold.

1) $\operatorname{spt}_{c_{1}}(d)=s p t_{c_{1}}\left(W_{1}\right)+s p t_{c_{1}}\left(W_{2}\right)+s p t_{c_{1}}\left(W_{3}\right)$

$$
=\frac{6}{16}=0.375 \text {. }
$$

2) $s p t_{c_{2}}(d)=s p t_{c_{2}}\left(W_{1}\right)+s p t_{c_{2}}\left(W_{2}\right)+s p t_{c_{2}}\left(W_{3}\right)$

$$
=\frac{5}{16}=0.313 \text {. }
$$

3) $s p t_{c_{3}}(d)=s p t_{c_{3}}\left(W_{1}\right)+s p t_{c_{3}}\left(W_{2}\right)+s p t_{c_{3}}\left(W_{3}\right)=0$

Remark 16. By Remark 14 and Proposition 3, we have the following conclusions.

1) The strength of coincident index (supporting monitoring signal) is 0.375 , which is maximal in strengths of condition attributes.

2) The strength of leading index (supporting monitoring signal) is 0.313 , which is less than the strength of coincident index and more than the strength of lagging index.

3) The strength of lagging index (supporting monitoring signal) is 0, which is minimum in strengths of condition attributes.

\section{Postscript}

In the end of this paper, we give some explanations

1) The investigation in this paper is conducted with a sample of 13 months for macro-economic climate index of China. The validity of the research conclusion and associated discussions is limited by the relatively small sample size. However, as stated earlier, results of this investigation will be helpful for Chinese government to make active macro-economic policy and to maintain the steady and relatively fast development of Chinese economic.
2) The investigation in this paper is based on partitions of the finite universe \$U\$ of discourse, but by using these partitions we are not able to solve neighboring question in numerical representations for some condition attributes. For example, leading index 98.86 is cooling and leading index 99.09 is stable in this paper. In recent years, the rough-set theory has been developed from partitions of the universe of discourse to covers of the universe of discourse $[14,15]$, which may provide a satisfactory solution for this neighboring question. Further exploratory might be performed towards this direction.

\section{References}

[1] National Bureau of Statistics of China, "Macro-Economic Climate Index of China from 2009-02 to 2010-05," May 2010. http://www.stats.gov.cn/english/

[2] G. Alvatore, M. Bentto and S. Roman, "Rough Set Theory for Multi Criteria Decision Analysis," European Journal of Operational Research, Vol. 129, No. 1, 2001, pp. 1-47. doi:10.1016/S0377-2217(00)00167-3

[3] C. Donna, "Artificial Interagency Research in Japan," Artificial Intelligence, Vol. 91, No. 1, 1997, pp. 122-129.

[4] A. Erbert, "Scientific Discovery and Simplicity of Method," Artificial Intelligence, Vol. 91, No. 2, 1997, pp. 177-181. doi:10.1016/S0004-3702(97)00019-2

[5] X. Ge and J. Qian, "Some Investigations on Higher Mathematics Scores for Chinese University Students," International Journal of Computer and Information Engineering, Vol. 3, No. 4, 2009, pp. 209-212.

[6] X. Ge, J. Li and Y. Ge, "Some Separations in Covering Approximation Spaces," International Journal of Computational and Mathematical Sciences, Vol. 4, No. 3, 2010, pp. 156-160.

[7] Z. Pawlak, "Rough Sets," International Journal of Computer and Information Sciences, Vol. 11, No. 5, 1982, pp. 341-356. doi:10.1007/BF01001956

[8] Z. Pawlak, "Rough Sets: Theoretical Aspects of Reasoning about Data," Kluwer Academic Publishers, Norwell, 1991.

[9] Z. Pawlak, "Rough Sets," Communications of ACM, Vol. 38, No. 11, 1995, pp. 89-95. doi:10.1145/219717.219791

[10] Z. Pawlak, "Vagueness and Uncertainty: A Rough Set Perspective," Computational Intelligence, Vol. 11, No. 2, 1995, pp. 227-232. doi:10.1111/j.1467-8640.1995.tb00029.x

[11] S. Padmini and H. Donald, "Vocabulary Mining for Information Retrieval:Rough Sets and Fuzzy Sets," Information Processing and Management, Vol. 37, No. 1, 2002, pp. 15-38.

[12] K. Qin, Y. Gao and Z. Pei, "On Covering Rough Sets," Lecture Notes in Artificial Intelligence, Vol. 4481, 2007, pp. 34-41.

[13] M. Stiefki and S. Smoliar, "What Computers Still Can'T Do: Five Reviews and a Response," Artificial Intelligence, 
Vol. 80, No. 1, 1996, pp. 95-97. doi:10.1016/0004-3702(95)00082-8

[14] M. Yahia, R. Mahmodr and N. Sulaimann, "Rough Neural Expert Systems," Expert System with Applications, Vol. 18, No. 2, 2002, pp. 87-99. doi:10.1016/S0957-4174(99)00055-X

[15] W. Zhang, W. Wu, J. Liang and D. Li, "Rough-Set The- ory and Its Method," Chinese Science Press, Beijing, 2002.

[16] W. Zhu and F. Wang, "On Three Types of Covering Rough Sets," IEEE Transactions on Knowledge and Data Engineering, Vol. 19, No. 8, 2007, pp. 1131-1144. doi:10.1109/TKDE.2007.1044 